\title{
Telepsiquiatría: una revisión sistemática cualitativa
}

\author{
ARIEL CASTRO $^{1}$, ALBERTO LARRAÍN $^{2}$, \\ ROSEMARIE FRITSCH ${ }^{3}$, GRACIELA ROJAS ${ }^{3}$
}

\section{Feasibility of implementing tele-psychiatry in Chile}

The objective of this review was to search the literature on the use of telemedicine in mental health and evaluate if it can play a role in Chile. A systematic, qualitative review was carried out to compile systematic reviews, meta-analysis, and clinical controlled trials (CCT) that were in English or Spanish and that applied information technologies for the treatment of psychiatric diseases. Excluded from the review were articles without summaries or articles that included only the trial design, without results. The references of each selected article were critically evaluated. Of the 265 articles found, 224 were excluded for failing to comply with the inclusion criteria. Therefore, 41 articles were left for analysis, 30 reporting CCT and 11 systematic reviews. It is concluded that the use of information technologies to provide mental health care is widespread. It can be implemented in geographically remote places, without access to specialized mental health care and be a part of complex interventions that integrate several components.

(Rev Med Chile 2012; 140: 789-796).

Key words: Mental health; Psychiatry; Telemedicine.

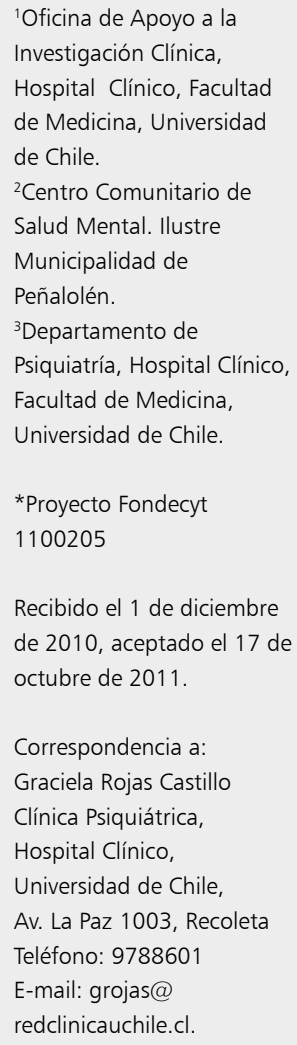

$\mathrm{E}$ n Chile, se ha impulsado la creación de programas de salud mental en el nivel primario de atención, el desarrollo de centros comunitarios de atención psiquiátrica $\mathrm{y}$, con la Reforma de Salud, se ha garantizado el acceso a la atención a las personas afectadas por esquizofrenia, depresión y consumo perjudicial de alcohol y drogas ${ }^{1}$. Estas medidas han entregado al equipo de salud primario un rol protagónico en el manejo de los trastornos mentales comunes y a los equipos especializados el manejo de los trastornos mentales graves.

De esta forma se ha enfrentado la altas tasas de prevalencia de trastornos mentales y el acceso inequitativo a la atención de salud mental, problemas que fueron demostrados por estudios epidemiológicos llevados a cabo en la década de 1990-992,3.

Los programas de tratamiento para los trastornos mentales que han demostrado efectividad son complejos e integran múltiples componentes -educación, farmacoterapia, intervenciones psicosociales, monitorización de los tratamientos-e involucran a una amplia gama de profesionales de la salud incluyendo la asesoría de profesionales especializados.

En el país, existe una distribución desigual de los recursos médicos especializados con una concentración en la zona central lo que, junto a nuestra geografía, dificulta el acceso equitativo a estos recursos ${ }^{4-7}$. Sin embargo, el país cuenta con un desarrollo importante de las telecomunicaciones y tecnología de información (TIC) las cuales pudiesen jugar un papel en el desafío que significa la resolución de problemas mentales y la provisión de servicios especializados ${ }^{8-10}$.

El propósito esta revisión es identificar y analizar literatura sobre el uso de la telepsiquiatría (TSQ) y evaluar si ésta puede jugar un papel en nuestro país. 


\section{Método}

Se llevó a cabo una búsqueda bibliográfica mediante tres modalidades: uso de bases de datos (Pubmed, Scielo, LILACS, OVID, ProQuest, Science Direct, EBSCO, Springer Link, Oxford Journals, The Cochrane Library), revistas científicas relevantes para el tema (Int J Methods Psychiatr Res, Psychiatr Serv, Telemedicine Journal, Telemedicine and $e$-Health) y listados de referencias de los artículos seleccionados.

Las palabras claves y las combinaciones fueron: [Telehealth OR Telemedicine OR Telepsychiatry] combinadas con [Mood Disorders OR Depression OR Depressive Disorder OR Schizophrenia OR Psychotic Disorders OR Substance Abuse OR SubstanceRelated Disorders OR Cocaine-Related Disorders OR Alcoholism OR Alcohol-Related Disorders OR Eating Disorders OR Bulimia Nervosa OR Bulimia OR Anxiety OR Anxiety Disorders OR Stress Disorders OR Post-Traumatic Stress OR Phobic Disorders OR Panic Disorder].

Se buscaron artículos que informasen sobre revisiones sistemáticas, metanálisis y ensayos clínicos controlados (ECC) que estuvieran en el idioma inglés o castellano y que aplicaran TICvideoconferencia, teléfono, plataformas informáticas e Internet -en salud mental para todas las patologías psiquiátricas y niveles de atención.

Se excluyeron aquellos artículos que no tuviesen un resumen o que publicasen el diseño de un estudio sin sus resultados.

Para cada uno de los artículos seleccionados se realizó una evaluación crítica de la literatura ${ }^{11}$.

\section{Resultados}

Se encontraron 265 artículos, se excluyeron 224 por no cumplir con los criterios de inclusión de la revisión, quedando 41 artículos: 30 sobre ECC y 11 revisiones sistemáticas (Figura 1).

\section{Características de los estudios}

De los 30 ECC, 27 fueron aleatorizados -de éstos sólo 18 describieron la forma de aleatorización-, 26 realizaron una comparación de las características basales de los sujetos -en 19 los pacientes no tenían diferencias al inicio de la intervención-y 19 utilizaron análisis de intención de tratamiento. La pérdida de los pacientes durante el seguimiento varió entre $1,27 \%$ y $57 \%$. El total de sujetos que participaron en los ECC seleccionados varió entre 12 y 600.

De las 11 revisiones sistemáticas incluidas en esta revisión, 10 describían objetivo y metodología, 7 comunicaban los criterios de selección de los estudios y 5 seleccionaron solamente ECC.

La TIC utilizadas en las intervenciones fueron teléfono y videoconferencia y se compararon con atención usual o con listas de espera.

\section{Estudios según patología}

Depresión

Los 13 artículos encontrados corresponden a 11 ECC que medían la efectividad de tratamientos para la depresión involucraron en mayor o menor medida la intervención de especialistas.

En dos de los estudios se implementaron programas colaborativos entre especialistas de salud mental y equipos o médicos no especialistas que usaban tecnologías para su comunicación ${ }^{12,13}$. El programa colaborativo de Fortney y col realizado en atención primaria, fue más efectivo que el tratamiento usual respecto a variables de salud mental y calidad de vida; adicionalmente tuvo mejor evaluación usuaria ${ }^{12}$. El programa colaborativo de Liu y col lograba mayor cantidad de días libres de depresión de las personas tratadas pero con un costo mayor ${ }^{13}$.

Otra modalidad de intervención utiliza las TIC como medio de intervención de los especialistas sobre los pacientes. Ruskin y cols llevaron a cabo un ECC que comparó un tratamiento para la depresión realizado por psiquiatras en forma presencial versus en forma remota, encontrando que ambos grupos mejoraban su sintomatología depresiva, no encontrando diferencias significativas entre ambos resultados de tratamientos ni tampoco respecto al uso de servicios, satisfacción usuaria ni $\operatorname{costos}^{14}$.

Un ECC con tres brazos comparó un tratamiento computarizado de orientación cognitivo conductual administrado en línea llamado "Coloree su vida", el manejo usual de la depresión llevado a cabo por un médico general y ambos juntos no encontrándose diferencias significativas a los seis meses entre estas tres posibilidades en relación a sintomatología depresiva y calidad de $\operatorname{vida}^{15}$; $\sin$ embargo, los análisis costo efectividad favorecían al tratamiento cognitivo conductual solo ${ }^{16}$. 


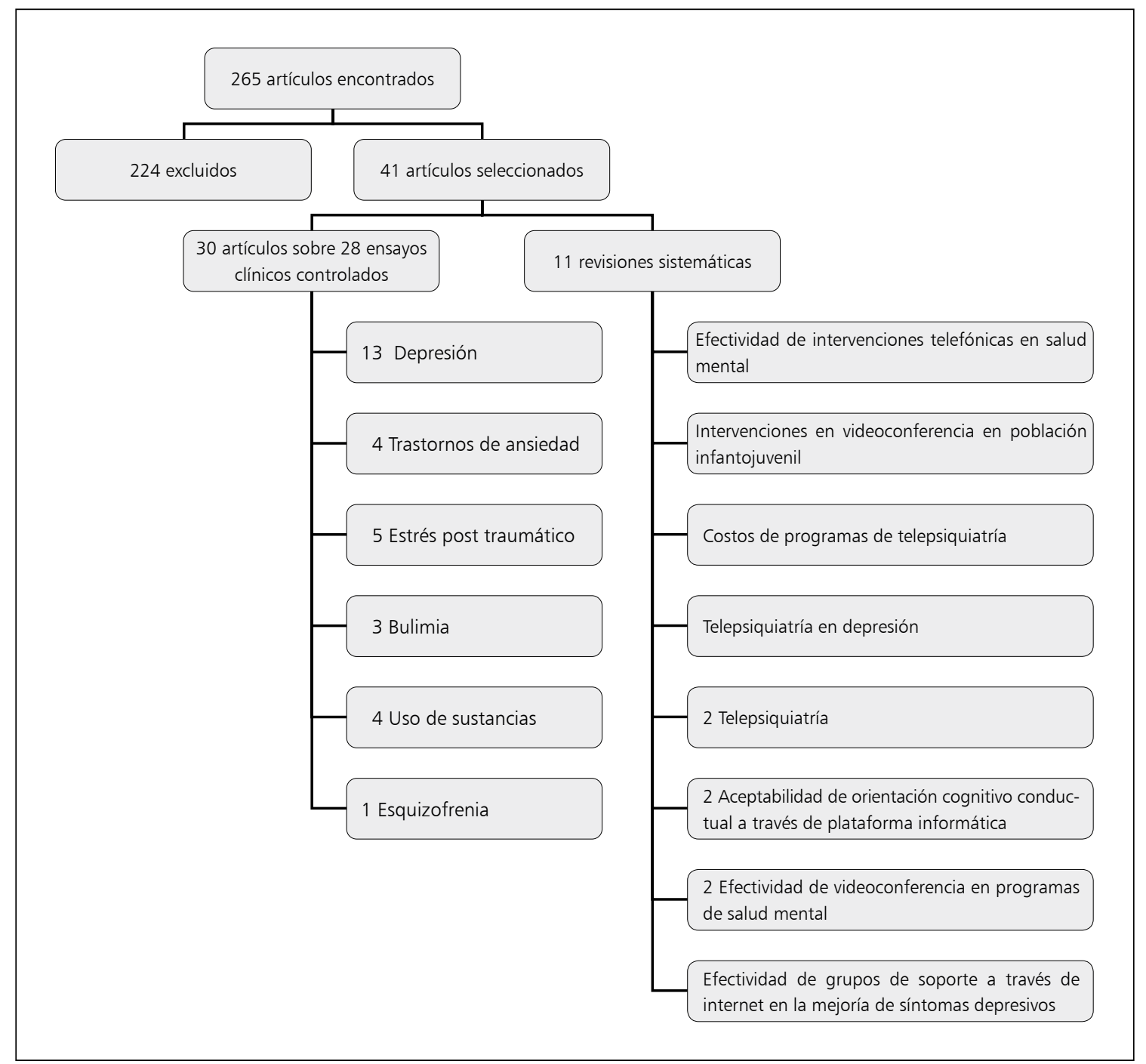

Figura 1. Diagrama de selección y caracterización de los estudios.

Cinco ECC prueban el uso del teléfono en el tratamiento de la depresión. Simon y cols comparan tres intervenciones: tratamiento usual en la atención primaria, éste más manejo telefónico de los casos con informe al médico tratante y ambos más psicoterapia cognitiva conductual administrada telefónicamente y concluyó que para pacientes que inician tratamiento farmacológico, esta última alternativa era la más efectiva y satisfactoria, seguida de la anterior y que el tratamiento usual era lo menos efectivo clínicamente ${ }^{17}$. El análisis económico indicó que el aumento de los costos del manejo telefónico más la psicoterapia era modesto a los 24 mese $^{18}$. El mismo autor llevó a cabo un ECC que midió la efectividad de farmacoterapia iniciada por psiquiatras con y sin monitoreo telefónico y concluyó que el monitoreo telefónico no mejoraba los resultados clínicos ${ }^{19}$. En Chile, Fritsch y cols llevaron a cabo un ECC que probó la efectividad a los tres y seis meses del monitoreo telefónico(TM) del tratamiento farmacoterapeutico en mujeres consultantes en consultorios primarios logrando el TM mayor adherencia a la farmacoterapia en comparación con el grupo que no fue monitoreado telefónicamente ${ }^{20}$. Un ECC llevado a cabo por Dietrich y cols comparó 
un programa estructurado de tratamiento de la depresión basado en criterios de mejoramiento de la calidad con monitoreo telefónico de casos versus tratamiento usual y encontró que el programa era efectivo y lograba mejores respuesta al tratamiento y mayor remisión de los síntomas a los seis meses ${ }^{21}$.

Tres ECC miden la efectividad de tratamientos para un cuadro depresivo comorbido con otras patologías. Rollman y cols midieron la efectividad de una intervención telefónica llevada a cabo por enfermeras supervisadas por un psiquiatra en colaboración con médicos de atención primaria de 8 meses de duración dirigido a pacientes con depresión post bypass coronario encontrando que a los 8 meses, la intervención telefónica era más efectiva en relación a la calidad de vida relacionada con la salud mental, a la sintomatología depresiva y a la funcionalidad física en comparación con el manejo usual de estos casos $^{22}$. Kay-Lambkin y cols llevaron a cabo un ECC de tres ramas en pacientes mayores de 16 años depresivos con consumo problemático de alcohol o marihuana quienes fueron aleatorizados luego de recibir una intervención breve (BI) a un grupo control, a un tratamiento de nueve sesiones de orientación cognitiva conductual persona a persona o computarizada con apoyo breve pero semanal persona a persona encontrando que el grupo control tiene resultados desfavorables en comparación con las dos intervenciones y que, a los 12 meses de seguimiento, ambas intervenciones fueron equivalentes en disminuir la sintomatología depresiva y el uso de substancias ahorrando la intervención computarizada tiempo del terapeuta ${ }^{23}$. Mohr y cols compararon dos tratamientos psicoterapéuticos administrados telefónicamente a pacientes que sufren de esclerosis múltiple y depresión encontrando que ambos logran reducir la sintomatología depresiva y que la psicoterapia de orientación cognitiva conductual logra resultados específicos mejores que la psicoterapia focal ${ }^{24}$.

García-Lizama y col llevaron a cabo una revisión sistemática entre los años 1997-2008 de ECC sobre uso de TSP en el tratamiento de la depresión concluyendo que aún no hay suficientes evidencias sobre su efectividad pero plantean que el tratamiento a través de videoconferencia pudiese obtener los mismos resultados que persona a persona y que plataformas de autoayuda podrían ayudar a pacientes en listas de espera para tratamientos o a pacientes en control ${ }^{25}$.
Una revisión sistemática informó buena aceptabilidad por parte de los beneficiarios de formas computarizadas de terapia cognitiva conductual para el tratamiento de la depresión informando que tendría buena acogida ${ }^{26}$. Otra revisión sistemática buscó el impacto de grupos de autoayuda por Internet en la sintomatología depresiva y encontró que los grupos de cáncer de mama reportaban una mayor disminución de la sintomatología depresiva que otro tipo de grupos ${ }^{27}$. Ambas revisiones señalan que faltan ensayos clínicos aleatorizados bien diseñados que entreguen mayor evidencia científica.

\section{Trastornos de ansiedad}

Ruwaard y cols llevaron a cabo un ECC que midió la efectividad de un tratamiento por Internet de orientación cognitiva conductual de siete módulos de 11 semanas de duración con supervisión de especialistas entrenados en pacientes con sintomatología crónica de trastorno de pánico y encontró que éstos mejoraron la psicopatología y la sintomatología de pánico en forma moderada o alta al término del tratamiento, con alta aceptación de éste y que, a los tres años, los resultados eran aun mejores ${ }^{28}$.

Calbring y cols midieron la efectividad de una intervención de autoayuda de diez módulos que se practicaba en una plataforma electrónica interactiva con soporte telefónico para pacientes con trastorno de pánico con o sin agarofobia, encontrando que $77 \%$ de los pacientes así tratados se recuperaban y que todos mejoraban las cogniciones, interpretaciones corporales, conductas evitativas, calidad de vida y niveles de ansiedad y depresión ${ }^{29}$. Ambos ECC tenían como grupo control una lista de espera.

Rollman y cols llevaron a cabo un ECC para medir la efectividad de un programa colaborativo que incluía manejo telefónico de casos con trastorno de pánico o de ansiedad generalizado de clínicas de atención primaria,resultando la intervención -manejo telefónico de casos por profesionales que psicoeducan, monitorean tratamiento e informan regularmente al equipo tratante-, más efectiva que el tratamiento usual en relación a reducción de sintomatología ansiosa, depresiva, calidad de vida y funcionalidad laboral a los doce meses de seguimiento ${ }^{30}$.

Un Programa Autoaplicado de Telepsicología para el tratamiento de fobias simples de animales pequeños usando escenarios virtuales de exposi- 
Telepsiquiatría: una revisión sistemática cualitativa - A. Castro et al

ción mostró mejoría sintomática a los tres meses de seguimiento ${ }^{31}$.

\section{Estrés post traumático (PTSD)}

Morland y cols midieron la efectividad de una terapia grupal para veteranos combatientes con PTSD por vídeo conferencia versus persona a persona y demostró resultados equivalentes en cuanto a manejo de la rabia a los seis meses de seguimiento $^{32}$.

Un ECC comparó la efectividad de una terapia de orientación cognitiva conductual de 16 a 25 semanas de duración para el PTSD entregada por videoconferencia versus persona a persona y no encontró diferencias significativas entre las dos modalidades respecto a frecuencia y severidad de los síntomas ${ }^{33}$.

Frueh y cols compararon la eficacia de una terapia cognitiva conductual de 14 semanas de duración para veteranos de guerra con PTSD por teleconferencia o persona a persona y tampoco encontraron diferencias significativas a los tres meses en cuanto a sintomatología clínica y satisfacción usuaria, pero el grupo persona a persona tuvo mejor adherencia al tratamiento ${ }^{34}$.

Knaevelsrud y cols midieron la efectividad de 10 sesiones de terapia cognitivo conductual entregada por psicólogos entrenados a través de internet y llevada a cabo en cinco semanas en pacientes con PTSD encontrando que ésta lograba mejoría sintomática específica y comórbida en comparación con el grupo control - pacientes de una lista de espera-a los tres meses de seguimiento logrando buena alianza terapéutica y poco abandono de tratamiento. El seguimiento a los 18 meses de estos pacientes reveló que la mejoría sintomática se mantenía ${ }^{35}$.

Litz y cols llevaron a cabo un ECC piloto que comparó dos tratamientos psicoterapéuticos entregados por Internet a pacientes con PTSD secundario a los ataques del 11 de septiembre al Pentágono o combatientes de la guerra de Iraq; un tratamiento consistía en autoayuda de orientación cognitivo conductual con apoyo de un terapeuta y el otro era una consejería de apoyo, encontrando que la primera era más efectiva en la reducción de los síntomas del PTSD y de síntomas depresivos y ansioso a los 6 meses de seguimiento ${ }^{36}$.

\section{Trastornos de la conducta alimentaria}

Ljotsson y cols midieron la efectividad de una intervención para pacientes adultos con bulimia nerviosa y atracones consistente en la entrega de un libro con material psicoeducativo y un programa de autoayuda de seis etapas con apoyo por internet para su ejecución, encontrando mejoría clínica significativa al término del tratamiento -doce semanas- la que se mantuvo a los seis meses $^{37}$. Fernández-Aranda y col midieron la efectividad de un tratamiento para bulimia nerviosa administrado por Internet que resultó ser apropiado para personas con sintomatología leve ${ }^{38}$. En ambos ECC el grupo control estaba constituido por pacientes de listas de espera. Mitchell y cols midieron la efectividad y aceptabilidad de un tratamiento psicoterapéutico manualizado de orientación cognitivo conductual de 20 sesiones para bulimia nerviosa administrado vía videoconferencia, encontrando que éste era aceptado por los pacientes y equivalente al mismo tratamiento administrado persona a persona ${ }^{39}$.

\section{Consumo de substancias}

Un ECC de tres ramas fue llevado a cabo por Mc Kay y cols en pacientes con dependencia a alcohol y/o cocaína quienes fueron aleatorizados luego de cumplir cuatro semanas de tratamiento ambulatorio intensivo encontrando que, a los dos años de seguimiento, los pacientes lograban tasas más altas de abstinencia si recibían, además de consejería y terapia grupal persona a persona, monitoreo telefónico semanal en comparación con terapias persona a persona -grupo de prevención de recaídas dos veces a la semana o terapia grupal dos veces a la semana- ; sin embargo, los pacientes de alto riesgo se beneficiaron más de la terapia grupal dos veces a la semana ${ }^{40}$.

Otro ECC evaluó una intervención -consejería telefónica breve- en pacientes con uso problemático de alcohol encontrando que ésta lograba disminuir el consumo riesgoso de alcohol a los seis meses de seguimiento en comparación con el grupo control ${ }^{41}$.

Kavanagh y cols llevaron a cabo un ECC con un diseño cross over -intervención inmediata versus tardía- para medir la efectividad de una intervención dirigida a adultos con Trastorno por uso de alcohol en atención primaria: los médicos generales recibían por correo información mensual sobre el estado de sus pacientes y éstos recibían por correo ocho cartas con material psicoeducativo de orientación cognitivo conductual sobre los riesgos del consumo problemático de 
alcohol así como autoevaluaciones encontrando que a los tres meses de seguimiento, la intervención temprana era más efectiva en disminuir la cantidad y frecuencia del consumo de alcohol, así como la sintomatología ansiosa y depresiva, pero a los 12 meses el consumo promedio de alcohol se mantenía alto ${ }^{42}$.

\section{Esquizofrenia}

En pacientes esquizofrénicos, un ECC comparó la efectividad de un programa de monitorización de adherencia a terapia antipsicótica a través de una plataforma informática versus el conteo de pastillas y autoreporte de adherencia y demostró que la primera es factible de implementar y tiene resultados equivalentes a la segunda en cuanto a adherencia al tratamiento ${ }^{43}$.

\section{Otros}

Se encontró una revisión sistemática sobre la efectividad de intervenciones telefónicas para enfermedades mentales que identificó 14 estudios que evaluaban intervenciones telefónicas para depresión, ansiedad, trastornos alimentarios, uso de sustancias y esquizofrenia y concluye que éstas pueden ser efectivas usando sesiones terapéuticas estructuradas y desarrollo de tareas ${ }^{44}$.

Se encontró también una revisión sistemática sobre telepsiquiatría infanto juvenil que concluye que la videoconferencia parece mejorar la accesibilidad a servicios de psiquiatría infanto juvenil y para fines educacionales ${ }^{45}$.

Ambas revisiones señalan la necesidad de mayor evidencia.

Una revisión sistemática sobre los costos de programas de TSQ señala que un factor importante a considerar es que ésta debe ser calculada sobre la demanda estimada para que sea financieramente viable en comparación con la atención usual por especialistas en salud mental ${ }^{46}$.

\section{Discusión}

Esta búsqueda encontró numerosos artículos sobre telepsiquiatría con calidad metodológica diversa. Hay alguna evidencia que intervenciones en salud mental llevadas a cabo a distancia pueden tener resultados similares a las realizadas persona a persona; sin embargo, los estudios encontrados tienen limitaciones metodológicas como la falta de un grupo de comparación, el número reducidos de sujetos incluidos en los estudios, la alta variabilidad en la definición y determinación de las variables para evaluar los resultados.

\section{Conclusiones}

El uso de TIC para entregar atención en salud mental a poblaciones es amplio y factible de implementar en lugares apartados geográficamente que no tienen acceso a la atención especializada en salud mental y como parte de intervenciones complejas que integran varios componentes.

La TSQ ha demostrado tener resultados equivalentes a la atención persona a persona, en diferentes niveles y ámbitos de atención en salud mental, con una buena aceptabilidad por parte de los usuarios, pero a un costo elevado. La incorporación de la tecnología en intervenciones complejas parece promisoria y es una posibilidad a evaluar en nuestro país que ha fomentado la implementación de programas de tratamiento que requieren trabajo colaborativo entre equipos de salud general y especialistas. En este sentido, parecen relevantes los resultados que pueda generar el proyecto de TSQ en curso en nuestro país que mide la efectividad de una colaboración entre psiquiatras y médicos generales para el tratamiento de la depresión, con el apoyo de una plataforma informática.

Sin embargo, se hace necesario evaluar los efectos de la TSQ sobre la transferencia y contratransferencia.

\section{Referencias}

1. Decreto Exento Ley $N^{\circ} 19.966$ Listado de Prestaciones Específicas, Ministerio de Salud.

2. Vicente B, Kohn R, Rioseco P, Saldivia S, Levav I,Torres $\mathrm{S}$, Lifetime and 12-month prevalence of DSM-III-R disorders in the Chile psychiatric prevalence study. Am J Psychiatry 2006; 163 (8): 1362-70.

3. Araya R, Lewis GH, Rojas G, Mann AH. "Patient knows best"-detection of common mental disorders in Santiago, Chile: cross sectional study. BMJ 2001; 322 (7278): 79-81.

4. Román $\mathrm{O}$, Acuña $\mathrm{M}$, Señoret $\mathrm{M}$, Disponibilidad de médicos en Chile al año 2004. Rev Med Chile 2006; 134: 1057-64.

5. Román O, Señoret M. Estado actual de las especialida- 
des médicas en Chile: realidad en el sistema público no municipalizado. Rev Med Chile 2008; 136: 99-106.

6. Skoknic Defilippis AM. Informe de la Comisión de Salud en Calidad de Investigadora de la Crisis Hospitalaria de la Salud Pública en Chile. Cámara de Diputados, Editor. 2008, Camara de Diputados, Valparaíso.

7. Banco Mundial, Gobierno de Chile, Estudio de Brechas de Oferta y Demanda de Médicos Especialistas en Chile, Departamento de Estudios de Recursos Humanos, Editor. 2010.

8. Digitales T. Estudio de Usos de Internet en Latinoamérica 2008. Disponible en: http://www.tendenciasdigitales. com/reporte_esp.asp?idr=121\&pg=71. Último Acceso [30 de mayo de 2009].

9. Digitales T. Estudio Usos de Internet en Latinoamérica 2009. Disponible en: http://www.tendenciasdigitales. com/reporte_esp.asp?idr $=121 \& p g=71$. Último Acceso [30 de mayo de 2009].

10. Capurro D. Health informatics in Chile: responding to health reforms. Health Info Libr J 2007; 24 (4): 287-91.

11. Sackett DL, Straus SE, Richardson WS, Rosenberg W, Haynes RB. Medicina basada en la evidencia. Cómo practicar y enseñar la MBE. 2001, Madrid: Elsevier.

12. Fortney J, Pyne J, Edlund M, Williams D, Robinson D, Mittal D, et al. A Randomized Trial of Telemedicinebased Collaborative Care for Depression. Journal of General Internal Medicine 2007; 22 (8): 1086-93.

13. Liu C, Hedrick S, Chaney E, Heagerty P, Felker B, Hasenberg $\mathrm{N}$, et al. Cost-effectiveness of collaborative care for depression in a primary care veteran population. Psychiatr Serv 2003; 54 (5): 698-704.

14. Ruskin PE, Silver-Aylaian M, Kling MA, Reed SA, Bradham DD, Hebel JR, et al. Treatment Outcomes in Depression: Comparison of Remote Treatment Through Telepsychiatry to In-Person Treatment. Am J Psychiatry 2004; 161(8): 1471-6.

15. De Graaf LE, Gerhards SA, Arntz A, Riper H, Metsemakers JF, Evers SM, et al. Clinical effectiveness of online computerised cognitive-behavioural therapy without support for depression in primary care: randomised trial. Br J Psychiatry 2009; 195 (1): 73-80.

16. Gerhards SA, De Graaf LE, Jacobs LE, Severens JL, Huibers MJ, Arntz A, et al. Economic evaluation of online computerised cognitive-behavioural therapy without support for depression in primary care: randomised trial. Br J Psychiatry 2010; 196: 310-8.

17. Simon GE, Ludman EJ, Tutty S, Operskalski B,Von Korff $\mathrm{M}$. Telephone psychotherapy and telephone care management for primary care patients starting antidepressant treatment: a randomized controlled trial. JAMA 2004; 292 (8): 935-42.
18. Simon G VKM, Rutter C, Wagner E. Randomised trial of monitoring, feedback, and management of care by telephone to improve treatment of depression in primary care. BMJ 2000; 320: 550-4.

19. Simon GE, Ludman EJ, Operskalski BH, Randomized trial of a telephone care management program for outpatients starting antidepressant treatment. Psychiatr Serv 2006; 57 (10): 1441-5.

20. Fritsch RAR, Solís J, Montt ME, Pilowsky D, Rojas G. Ensayo clínico randomizado de farmacoterapia con monitorización telefónica para mejorar el tratamiento de la depresión en la atención primaria en Santiago, Chile. Rev Med Chile 2007; 135: 587-95.

21. Dietrich AJ, Oxman TE, Williams JW, Jr. Schulberg HC, Bruce ML, Lee PW, et al. Re-engineering systems for the treatment of depression in primary care: cluster randomised controlled trial. BMJ 2004; 329 (7466): 602.

22. Rollman BL, Belnap BH, Lemenager MS, Mazumdar S, Houck PR, Counihan PJ, et al. Telephone-delivered collaborative care for treating post-CABG depression: a randomized controlled trial. JAMA 2009; 302 (19): 2095-103.

23. Kay-Lambkin FJ, Baker AL, Lewin TJ, Carr VJ. Computer-based psychological treatment for comorbid depression and problematic alcohol and/or cannabis use: a randomized controlled trial of clinical efficacy. Addiction 2009; 104 (3): 378-88.

24. Mohr DC, Hart SL, Julian L, Catledge C, Honos-Webb L, Vella L, et al. Telephone-administered psychotherapy for depression. Arch Gen Psychiatry 2005; 62 (9): 1007-14.

25. García-Lizana F, Muñoz-Mayorga I. Telemedicine for depression: a systematic review. Perspect Psychiatr Care 2010; 46 (2): 119-26.

26. Kaltenthaler E, Sutcliffe P, Parry G, Beverley C, Rees A, Ferriter M. The acceptability to patients of computerized cognitive behaviour therapy for depression: a systematic review. Psychol Med 2008; 38 (11): 1521-30.

27. Griffiths KM, Calear AL, Banfield M. Systematic review on Internet Support Groups (ISGs) and depression (1): Do ISGs reduce depressive symptoms? J Med Internet Res 2009; 11 (3): e40.

28. Ruwaard J, Broeksteeg J, Schrieken B, Emmelkamp P, Lange A. Web-based therapist-assisted cognitive behavioral treatment of panic symptoms: a randomized controlled trial with a three-year follow-up. J Anxiety Disord 2010; 24 (4): 387-96.

29. Carlbring P, Bohman S, Brunt S, Buhrman M, Westling BE, Ekselius L, et al. Remote treatment of panic disorder: a randomized trial of internet-based cognitive behavior therapy supplemented with telephone calls. Am J Psychiatry 2006; 163 (12): 2119-25. 
30. Rollman BL, Belnap BH, Mazumdar S, Houck PR, Zhu F, Gardner W, et al. A Randomized Trial to Improve the Quality of Treatment for Panic and Generalized Anxiety Disorders in Primary Care. Arch Gen Psychiatry 2005; 62 (12): 1332-41.

31. Botella C, Quero S, Banos RM, García-Palacios A, Breton-López J, Alcaniz M, et al. Telepsychology and self-help: the treatment of phobias using the internet. Cyberpsychol Behav 2008; 11 (6): 659-64.

32. Morland LA, Greene CJ, Rosen CS, Foy D, Reilly P, Shore $\mathrm{J}$, et al. Telemedicine for anger management therapy in a rural population of combat veterans with posttraumatic stress disorder: a randomized noninferiority trial. J Clin Psychiatry, 2010.

33. Germain V, Marchand A, Bouchard S, Drouin MS, Guay S. Effectiveness of cognitive behavioural therapy administered by videoconference for posttraumatic stress disorder. Cogn Behav Ther 2009; 38 (1): 42-53.

34. Frueh B, Monnier J, Yim E, Grubaugh A, Hamner M, Knapp R. A randomized trial of telepsychiatry for posttraumatic stress disorder. J Telemed Telecare 2007; 13 (3): 142-7.

35. Knaevelsrud C, Maercker A. Internet-based treatment for PTSD reduces distress and facilitates the development of a strong therapeutic alliance: a randomized controlled clinical trial. BMC Psychiatry 2007; 7: 13.

36. Litz BT, Engel CC, Bryant RA, Papa A. A randomized, controlled proof-of-concept trial of an Internet-based, therapist-assisted self-management treatment for posttraumatic stress disorder. Am J Psychiatry 2007; 164 (11): 1676-83.

37. Ljotsson B, Lundin C, Mitsell K, Carlbring P, Ramklint $\mathrm{M}$, Ghaderi A. Remote treatment of bulimia nervosa and binge eating disorder: a randomized trial of Internetassisted cognitive behavioural therapy. Behav Res Ther
2007; 45 (4): 649-61.

38. Fernández-Aranda F, Núñez A, Martínez C, Krug I, Cappozzo M, Carrard I, et al. Internet-based cognitivebehavioral therapy for bulimia nervosa: a controlled study. Cyberpsychol Behav 2009; 12 (1): 37-41.

39. Mitchell JE, Crosby RD, Wonderlich SA, Crow S, Lancaster $\mathrm{K}$, Simonich $\mathrm{H}$, et al. A randomized trial comparing the efficacy of cognitive-behavioral therapy for bulimia nervosa delivered via telemedicine versus face-to-face. Behav Res Ther 2008; 46 (5): 581-92.

40. Mckay JR, Lynch KG, Shepard DS, Pettinati HM. The Effectiveness of Telephone-Based Continuing Care for Alcohol and Cocaine Dependence: 24-Month Outcomes. Arch Gen Psychiatry 2005; 62 (2): 199-207.

41. Eberhard S, Nordstrom G, Hoglund P, Ojehagen A. Secondary prevention of hazardous alcohol consumption in psychiatric out-patients: a randomised controlled study. Soc Psychiatry Psychiatr Epidemiol 2009; 44 (12): 1013-21.

42. Kavanagh D, Connolly JM. Mailed treatment to augment primary care for alcohol disorders: a randomised controlled trial. Drug Alcohol Rev 2009; 28 (1): 73-80.

43. Frangou S, Sachpazidis I, Stassinakis A, Sakas G. Telemonitoring of medication adherence in patients with schizophrenia. Telemed J E Health 2005; 11 (6): 675-83.

44. Leach LS,Christensen H. A systematic review of telephone-based interventions for mental disorders. J Telemed Telecare, 2006. 12(3): 122-9.

45. Pesämaa L, Ebeling H, Kuusimäki M-L, Winblad I, et al. Videoconferencing in child and adolescent telepsychiatry: a systematic review of the literature. Journal of Telemedicine and Telecare 2004; 10 (4): 187.

46. Hyler SE, Gangure DP. A review of the costs of telepsychiatry. Psychiatr Serv 2003; 54 (7): 976-80. 